REGARDS

SUR LEECONOMIE ALLEMANDE

BULLETIN ECONOMIQUE DU CIRAC

\section{Regards sur l'économie allemande}

Bulletin économique du CIRAC

$89 \mid 2008$

Varia

\title{
Finances publiques : l'équilibre reporté à 2013 ?
}

Isabelle Bourgeois

\section{OpenEdition}

\section{Journals}

Édition électronique

URL : http://journals.openedition.org/rea/2863

DOI : $10.4000 /$ rea.2863

ISBN : 978-2-8218-0874-4

ISSN : 1965-0787

Éditeur

CIRAC

Édition imprimée

Date de publication : 1 décembre 2008

Pagination : 35-37

ISSN : 1156-8992

\section{Référence électronique}

Isabelle Bourgeois, «Finances publiques : l'équilibre reporté à 2013 ? ", Regards sur l'économie allemande [En ligne], 89 | décembre 2008, mis en ligne le 01 décembre 2010, consulté le 15 septembre 2020. URL : http://journals.openedition.org/rea/2863

Ce document a été généré automatiquement le 15 septembre 2020

(C) CIRAC 


\title{
Finances publiques : l'équilibre reporté à 2013 ?
}

\author{
Isabelle Bourgeois
}

Ces dernières années, l'Allemagne a fait «de grands progrès en matière de consolidation budgétaire ", se félicite le Conseil des Sages dans son rapport annuel 2008/09. Depuis 2003 , le déficit a pu être ramené de $-4,0 \%$ à $-0,1 \%$ en 2008 . Dans le même temps, le ratio de la dette publique est passé de $65,1 \%$ à $63,4 \%$ du PIB ; il reste néanmoins encore supérieur aux 60 \% fixé par le Pacte de Stabilité et de Croissance.

Evolution 2005-2008 du budget global de la RFA

(en milliards $€$ et évolution en \% par rapport à l'année précédente)

\begin{tabular}{|l|l|l|l|l|l|l|l|l|}
\hline & $\mathbf{2 0 0 5}$ & évol. & $\mathbf{2 0 0 6}$ & évol. & $\mathbf{2 0 0 7}$ & évol. & $\mathbf{2 0 0 8}{ }^{\text {a) }}$ & évol. \\
\hline & $\mathbf{9 7 6 , 2}$ & $+\mathbf{1 , 9}$ & $\mathbf{1} \mathbf{0 1 6 , 4}$ & $+\mathbf{4 , 1}$ & $\mathbf{1} \mathbf{0 6 5 , 9}$ & $\mathbf{+ 4 , 9}$ & $\mathbf{1} \mathbf{0 9 1 , 6}$ & $+\mathbf{2 , 4}$ \\
\hline Impôts, dont & 493,2 & $+2,5$ & 530,5 & $+7,6$ & 576,3 & $+8,6$ & 593,1 & $+2,9$ \\
\hline directs & 227,6 & $+2,9$ & 250,1 & $+9,9$ & 270,8 & $+8,3$ & 286,5 & $+5,8$ \\
\hline indirects & 265,6 & $+2,1$ & 280,5 & $+5,6$ & 305,5 & $+8,9$ & 306,6 & $+0,4$ \\
\hline Cotisations sociales & 396,5 & 0,0 & 399,9 & $+0,9$ & 399,9 & 0,0 & 407,5 & $+1,9$ \\
\hline Cessions, subventions, revenus du \\
patrimoine
\end{tabular}




\begin{tabular}{|l|l|l|l|l|l|l|l|l|}
\hline Rémunérations et salaires & 168,7 & $-0,5$ & 167,5 & $-0,7$ & 168,0 & $+0,3$ & 173,2 & $+3,1$ \\
\hline Intérêts & 62,3 & $-0,1$ & 65,3 & $+4,7$ & 67,4 & $+3,2$ & 70,0 & $+3,9$ \\
\hline Subventions & 27 & $-4,7$ & 27 & $-2,3$ & 27 & $+1,4$ & 27 & $+0,2$ \\
\hline Prestations sociales & 429,6 & $+0,2$ & 426,2 & $-0,8$ & 418,4 & $-1,8$ & 421,6 & $+0,8$ \\
\hline Prestations sociales en nature & 167,5 & $+2,5$ & 171,8 & $+2,5$ & 178,3 & $+3,8$ & 185,4 & $+4,0$ \\
\hline Autres transferts courants & 35,5 & $+6,8$ & 35,3 & $-0,5$ & 36,4 & $+3,0$ & 39,4 & $+8,2$ \\
\hline \hline Transferts de patrimoine b) & 34,6 & $+2,3$ & 30,5 & $-12,0$ & 38,5 & $+26,4$ & 34,5 & $-10,5$ \\
\hline Investissements bruts & 31,0 & $-2,3$ & 32,6 & $+5,3$ & 35,6 & $+9,2$ & 37,7 & $+6,0$ \\
\hline Autres & $-1,4$ & - & $-1,4$ & - & $-1,4$ & - & $-1,5$ & - \\
\hline Solde budgétaire b) & $-\mathbf{7 4 , 3}$ & - & $\mathbf{- 3 5 , 9}$ & - & $\mathbf{- 4 , 2}$ & - & $\mathbf{- 1 , 5}$ & - \\
\hline Déficit (en \% du PIB) & $\mathbf{- 3 , 3}$ & - & $\mathbf{- 1 , 5}$ & - & $\mathbf{- 0 , 2}$ & - & $\mathbf{- 0 , 1}$ & - \\
\hline
\end{tabular}

Source des données : Sachverständigenrat zur Begutachtung der gesamtwirtschaftlichen Entwicklung, "Die Finanzkrise meistern - Wachstumskräfte stärken », rapport annuel 2008/09, novembre 2008, p. 197. a) estimation ; b) les estimations 2008 ne tiennent pas compte des répercussions possibles de la Loi de stabilisation des marchés financiers entrée en vigueur le 17 octobre ; c) éducation, défense...

\section{Cap de consolidation budgétaire depuis 2003}

2 Mais au grand regret du Conseil des Sages, ces progrès ont été réalisés surtout par une hausse des recettes fiscales, et bien moins par une baisse des dépenses. Car une consolidation obtenue par l'augmentation des recettes "s'avère en règle générale moins durable " que si elle avait été effectuée par une réduction des dépenses, expliquent les Sages en rappelant l'analyse qu'ils avaient déjà formulée dans leur rapport de 2003/04. En outre, une telle stratégie comporte en germe le risque que, une fois le déficit réduit, "se créée une nouvelle spirale de la convoitise ". S'il faut œuvrer à la soutenabilité des finances publiques, c'est pour engranger, en période de croissance, la marge de manœuvre qui permet, en période de récession, "d'inscrire dans la durée, voire de développer les catégories de dépenses favorables à une croissance sur le long terme", autrement dit: d'investir dans les domaines porteurs d'avenir pour renforcer la compétitivité globale du site. L'Allemagne s'est bien engagée sur cette voie depuis 2003.

\section{Mais toujours trop de dépenses dans le domaine social}

3 La marge de manœuvre n'en reste pas moins limitée, étant donné que, en 2008, les dépenses publiques ont augmenté de 2,1\%, tirées surtout par les budgets sociaux (dépenses de médicaments, financement des hôpitaux, assurance dépendance), en 
hausse continue depuis plusieurs années. Les prestations d'intérêt général ont crû elles aussi, reflet du renchérissement des coûts de l'énergie et de l'effort budgétaire consenti à l'éducation ainsi qu'à la défense. Et l'année 2008 a signifié une rupture avec la modération salariale observée au cours des années passées dans la fonction publique : dans les Länder, les fonctionnaires et salariés du public ont vu leur rémunération augmenter de $2,9 \%$, dans les communes et au niveau du Bund, de quelque $5 \%$ dans l'ensemble.

4 Enfin, malgré l'amélioration continue du marché de l'emploi qui a réduit le volume des transferts publics aux chômeurs, les prestations sociales ont été en hausse de $0,8 \%$ en $2008 \mathrm{du}$ fait de l'entrée en vigueur de diverses mesures fiscales, dont l'allongement de la durée de versement des indemnités chômage aux seniors, la hausse des pensions de retraite, l'augmentation des aides à la formation professionnelle ou celle des allocations parentales. Ces mesures avaient été prises que le gouvernement "n'avait pas pu se fermer aux récents débats sur le thème: 'la croissance ne profite pas à tous les citoyens'», et donc cédé à la tentation de redistribuer les richesses, regrettent les Sages.

\section{La bonne tenue de l'activité et de l'emploi a fait progresser les recettes}

5 La progression des recettes s'est poursuivie en 2008, la bonne tenue de l'activité se traduisant surtout par une augmentation de l'encours fiscal, principalement du produit des impôts directs. Le produit de la TVA ne s'est guère accru, la consommation étant restée flageolante en 2008. A noter que, pour la première fois, cette année, la vente de droits d'émissions ('droits à polluer') a rapporté 0,9 milliard $€$. Si le produit des cotisations sociales a augmenté de $1,9 \%$ en 2008 malgré la diminution des cotisations chômage (ramenées de 4,2\% du salaire brut à $3,3 \%$ ), cela est dû essentiellement à la bonne tenue de l'emploi : 540000 emplois soumis à cotisations sociales ont en effet été créés.

Evolution 2005-2008 de l'encours fiscal (principales catégories d'impôts) en RFA (en milliards $€$ et évolution en \% par rapport à l'année précédente)

\begin{tabular}{|l|l|l|l|l|l|l|l|l|}
\hline & $\mathbf{2 0 0 5}$ & évol. & $\mathbf{2 0 0 6}$ & évol. & $\mathbf{2 0 0 7}$ & évol. & $\begin{array}{l}\mathbf{2 0 0 8} \\
\text { a) }\end{array}$ & évol. \\
\hline \hline Impôt sur le revenu & 172,3 & $+1,8$ & 193,9 & $+12,5$ & 217,0 & $+12,0$ & 234,2 & $+7,9$ \\
\hline Impôt sur le revenu des salariés & 118,9 & $-4,0$ & 122,6 & $+3,1$ & 131,8 & $+7,5$ & 142,2 & $+7,9$ \\
\hline Impôt sur le revenu des non salariés & 9,8 & $+81,1$ & 17,6 & $+79,9$ & 25,0 & $+42,5$ & 32,0 & $+28,0$ \\
\hline Impôts sur les plus values & 10,0 & $+0,3$ & 11,9 & $+19,6$ & 13,8 & $+15,9$ & 15,7 & $+14,0$ \\
\hline Impôt sur le paiement des intérêts & 7,0 & $+3,2$ & 7,6 & $+9,2$ & 11,2 & $+46,5$ & 12,7 & $+13,3$ \\
\hline Impôt sur les bénéfices des sociétés & 16,3 & $+24,5$ & 22,9 & $+40,0$ & 22,9 & $+0,3$ & 18,4 & $-19,8$ \\
\hline Taxe de solidarité & 10,3 & $+2,1$ & 11,3 & $+9,4$ & 12,3 & $+9,4$ & 13,2 & $+6,5$ \\
\hline
\end{tabular}




\begin{tabular}{|l|l|l|l|l|l|l|l|l|}
\hline $\begin{array}{l}\text { Impôt sur les bénéfices des professions } \\
\text { industrielles et commerciales }\end{array}$ & 32,1 & $+13,2$ & 38,4 & $+19,4$ & 40,1 & $+4,5$ & 39,5 & $-1,6$ \\
\hline TVA & 139,7 & $+1,7$ & 146,7 & $+5,0$ & 169,9 & $+15,6$ & 175,0 & $+3,2$ \\
\hline Taxe sur l'énergie & 40,1 & $-4,0$ & 39,9 & $-0,5$ & 39,0 & $-2,4$ & 39,2 & $+0,6$ \\
\hline Taxe sur l'électricité & 6,5 & $-2,0$ & 6,3 & $-2,9$ & 6,4 & $+1,3$ & 6,0 & $-5,6$ \\
\hline \hline Taxe sur le tabac & 14,3 & $+4,7$ & 14,4 & $+0,8$ & 14,3 & $-0,9$ & 13,4 & $-6,3$ \\
\hline Taxe sur les assurances & 8,8 & 0,0 & 8,8 & $+0,3$ & 10,3 & $+17,7$ & 10,4 & $+0,7$ \\
\hline \hline Taxe sur les véhicules & 8,7 & $+12,1$ & 8,9 & $+3,0$ & 8,9 & $-0,4$ & 8,9 & $-0,2$ \\
\hline Droit de mutation des biens immobiliers & 4,8 & $+3,1$ & 6,1 & $+27,8$ & 7,0 & $+13,5$ & 96,0 & $-14,2$ \\
\hline Impôt sur les successions & 4,1 & $-4,4$ & 3,8 & $-8,2$ & 4,2 & $+11,7$ & 5,0 & $+18,0$ \\
\hline [ण Encours fiscal & $\mathbf{4 5 2 , 1}$ & $+2, \mathbf{1}$ & $\mathbf{4 8 8 , 4}$ & $+\mathbf{+ 8 , 0}$ & $\mathbf{5 3 8 , 2}$ & $+\mathbf{1 0 , 2}$ & $\mathbf{5 5 9 , 2}$ & $+3,9$ \\
\hline \hline Taux de prélèvement brut en \% du PIB & $\mathbf{2 0 , 2}$ & - & $\mathbf{2 1 , 0}$ & - & $\mathbf{2 2 , 2}$ & - & $\mathbf{2 2 , 4}$ & - \\
\hline Taux de prélèvement net en \% du PIB b) & $\mathbf{2 2 , 3}$ & - & $\mathbf{2 3 , 0}$ & - & $\mathbf{2 4 , 0}$ & - & $\mathbf{2 4 , 1}$ & - \\
\hline
\end{tabular}

Source des données : Sachverständigenrat zur Begutachtung der gesamtwirtschaftlichen Entwicklung, "Die Finanzkrise meistern - Wachstumskräfte stärken », rapport annuel 2008/09, novembre 2008, p. 199. a) estimation ; b) taux net : encours fiscal + prime à l'investissement (IR et IS) + prime d'accession à la propriété + allocations parentales + prime assurance vieillesse.

6 La bonne tenue de l'emploi, conjuguée aux hausses salariales, a fait aussi progresser de $7,9 \%$ le produit de l'impôt sur le revenu des salariés en 2008. Quant à la forte hausse (28\%) du produit de l'impôt sur le revenu non salarié, elle s'explique largement par la suppression de la prime d'accession à la propriété et par l'entrée en vigueur du nouveau barème plafond de $45 \%$ qui s'applique aux particuliers comme aux patronspropriétaires de PME. La bonne tenue passée de l'activité se reflétant dans le volume des dividendes versés aux actionnaires, elle a également continué à gonfler l'encours de l'impôt sur les plus values (14\%). Le recul du produit de l'impôt sur les sociétés comme de l'impôt sur les bénéfices des professions industrielles et commerciales a pour principale raison l'entrée en vigueur cette année de la réforme de la fiscalité des entreprises. Depuis l'été s'y ajoute cependant une tendance à la contraction des bénéfices, un des premiers effets de la crise financière sur l'économie réelle.

7 Au total, observe le Conseil des Sages, la structure de l'encours fiscal tend à se modifier au profit des impôts directs parce que l'évolution décevante de la consommation a limité l'impact potentiel de la hausse de trois points du montant de la TVA intervenue en 2007. 


\section{Seul le Bund est déficitaire en 2008}

8 Si on considère non plus le budget global de la RFA, mais sa structure par ensembles, on s'aperçoit que seul le Bund est déficitaire en 2008 - les Länder, les communes et les caisses de protection sociale étant excédentaires.

\section{Le budget des Länder est excédentaire...}

En 2008, les recettes des Länder ont augmenté de 2,5\%, du fait notamment de la hausse des impôts communs (quelque $70 \%$ des recettes fiscales sont partagées entre les différents échelons territoriaux) et des impôts qui leur reviennent en propre, comme l'impôt sur les successions et les donations. Pourtant, ils ont vu progresser de 3,8\% leurs dépenses sociales (départs massifs à la retraite dans leur fonction publique), et certains d'entre eux ont dû également financer le sauvetage de leurs banques publiques (Bavière, Rhénanie du Nord-Westphalie et Saxe). Dans l'ensemble, les Länder récoltent depuis deux ans les fruits de leur politique de consolidation budgétaire, ce qui explique aussi, soit dit en passant, le peu d'enthousiasme qu'ils mettent à conclure un Pacte de stabilité interne à la RFA dans le cadre de la réforme du fédéralisme financier.

Evolution des soldes budgétaires en RFA (Bund, Länder, communes, protection sociale) *)

\begin{tabular}{|c|c|c|c|c|c|c|c|c|}
\hline & \multicolumn{2}{|l|}{2005} & \multicolumn{2}{|l|}{2006} & \multicolumn{2}{|l|}{2007} & \multicolumn{2}{|l|}{$2008^{\text {a) }}$} \\
\hline & mrds. $€$ & $\begin{array}{ll}\% & d u \\
\text { PIB } & \end{array}$ & mrds. $€$ & $\begin{array}{ll}\% & d u \\
\text { PIB } & \end{array}$ & mrds. $€$ & $\begin{array}{ll}\% & d u \\
\text { PIB } & \end{array}$ & mrds. $€$ & $\begin{array}{ll}\% & d u \\
P I B & \end{array}$ \\
\hline $\begin{array}{l}\text { Collectivités } \\
\text { territoriales : }\end{array}$ & $-70,2$ & $-3,1$ & $-40,9$ & $-1,8$ & $-14,6$ & $-0,6$ & $-8,9$ & $-0,4$ \\
\hline - Bund & $-47,7$ & $-2,1$ & $-34,6$ & $-1,5$ & $-26,2$ & $-1,1$ & $-18,8$ & $-0,8$ \\
\hline - Länder & $-22,6$ & $-1,0$ & $-10,3$ & $-0,4$ & $+3,4$ & 0,1 & $+1,1$ & 0,0 \\
\hline - communes & $+0,1$ & 0,0 & $+4,0$ & 0,2 & $+8,3$ & 0,3 & $+8,6$ & 0,3 \\
\hline Protection sociale & $-4,1$ & $-0,2$ & $+5,0$ & 0,2 & $+10,4$ & 0,4 & $+7,4$ & 0,3 \\
\hline 蔵 RFA & $-74,3$ & $-3,3$ & $-35,9$ & $-1,5$ & $-4,2$ & $-0,2$ & $-1,5$ & $-0,1$ \\
\hline
\end{tabular}

Source des données : Sachverständigenrat zur Begutachtung der gesamtwirtschaftlichen Entwicklung, "Die Finanzkrise meistern - Wachstumskräfte stärken », rapport annuel 2008/09, novembre 2008, p. 206. *) hors produit des privatisations; a) estimation.

\section{... de même que celui des communes}

$10 \mathrm{Si}$, malgré un léger recul du produit de la taxe sur les bénéfices des professions industrielles et commerciales qui leur revient en propre, et malgré la hausse de dépenses en matière de transferts (aide sociale) et de salaires (services communaux), 
les communes ont vu globalement leurs budgets rester excédentaires, c'est qu'elles profitent elles aussi de la hausse du volume de l'impôt sur les revenus salariés, un impôt commun. De même, comme leurs budgets sont dans l'ensemble équilibrés depuis 2005, elles ont vu également se réduire le poids du service de la dette.

\section{La bonne tenue de l'activité a renfloué les caisses de protection sociale}

11 Quant à l'excédent des caisses de protection sociale, il est dû lui aussi à la bonne tenue de l'activité ; du fait la hausse des revenus salariaux, le versement complémentaire du Bund aux caisses du régime légal des retraites s'est légèrement accru également. Ces recettes compensent l'abaissement de 0,9 point de pourcentage du taux de cotisation chômage, partiellement corrigé par une augmentation de 0,1 point en moyenne des taux de cotisation à l'assurance maladie légale et, depuis juillet, celle de 0,25 point de la cotisation dépendance. Dans l'ensemble, la hausse des dépenses (prolongement de la durée de versement des allocations chômage aux seniors, hausse des pensions de retraite, forte augmentation des dépenses de santé) a pu être compensé par la nette progression de l'emploi soumis à cotisations sociales.

\section{Le Bund contraint de creuser le déficit dans sa loi de programmation budgétaire 2009}

12 La récession qui frappe maintenant l'Allemagne met à mal le cercle vertueux qui avait commencé à porter ses fruits et repousse au-delà de 2011 l'échéance de l'équilibre budgétaire global. La loi de programmation budgétaire 2009 du Bund, adoptée par le Bundestag le 28 novembre, porte à 290 milliards $€$ les dépenses de cet échelon territorial (1,6 milliard de plus que dans le budget prévisionnel), soit une hausse de 6,8 milliards $€$ par rapport à l'état prévisionnel des dépenses 2008. Les recettes (271,5 milliards $€$ ) ont été revues à la baisse (6,4 milliards $€$ de moins) par rapport à la version initiale du texte qui avait été soumise aux députés avant que se confirme l'entrée en récession. L'encours fiscal ne devrait en effet plus s'établir qu'à 244,1 milliards $€$ (4,6 milliards $€$ de moins que prévu). Le montant du nouvel endettement (18,5 milliards $€$ au lieu de 11,5 milliards $€$ ) est compatible avec la Constitution, puisqu'il reste inférieur aux investissements, fixés à 11,5 milliards $€$ $(+1,3$ milliard $€)$. Le budget adopté par le Bundestag est établi sur l'hypothèse d'une contraction du PIB comprise entre $-0,2 \%$ à $-1 \%$. Il sera probablement révisé encore au début 2009, quand le gouvernement fédéral aura pu intégrer les données du $4^{\mathrm{e}}$ trimestre 2008 (avec un PIB en net recul, selon les premières estimations) dans le rapport de politique économique qu'il présentera fin janvier. (IB) 
INDEX

Mots-clés : budget, déficit, dépenses, finances publiques, fiscalité, impôt, Länder, politique budgétaire, politique économique, politique sociale, région 